\title{
Portomesenteric Vein Thrombosis After Laparoscopic Sleeve Gastrectomy: Incidence, Analysis and Follow-Up in 1236 Consecutive Cases
}

\author{
Rodrigo Villagrán $^{1}$ • Gabriela Smith ${ }^{1}$ - Walter Rodriguez ${ }^{2}$ • Carlos Flores $^{1}$ • \\ Mario Cariaga $^{1}$ - Sofía Araya ${ }^{1}$ - Marisol Yañez ${ }^{1}$ Paulina Fuentes ${ }^{1,4}$. \\ Jeannette Linares ${ }^{3,4} \cdot$ Antonio Zapata $^{3}$
}

Published online: 14 April 2016

(C) The Author(s) 2016. This article is published with open access at Springerlink.com

\begin{abstract}
Background Portomesenteric vein thrombosis (PMVT) is a rare but severe complication after laparoscopic bariatric surgery, with potentially serious consequences. We aimed to describe the incidence, clinical features, management, outcome, and midterm follow-up in patients with PMVT after laparoscopic sleeve gastrectomy (LSG).

Methods This retrospective and descriptive study included patients who underwent LSG between November 2009 and July 2015 and developed PMVT. The following data were analyzed: age, gender, body mass index (BMI), thrombosis risk factors, surgical technique, thromboembolic prophylaxis, primary surgery outcomes, clinical features, treatment, thrombophilia testing results, and follow-up findings, including imaging and endoscopic findings.

Results A total of 1236 patients underwent LSG, and 5 $(0.4 \%)$ developed PMVT. The mean age was 34.4 years, and 3 patients were women. The mean BMI was $38.5 \mathrm{~kg} /$ $\mathrm{m}^{2}$. Two patients had received hormonal contraceptive treatment. Four patients had a history of smoking. All of the patients received anticoagulant treatment, and none required surgery. The mean hospitalization duration was 7.6 days. Two
\end{abstract}

Rodrigo Villagrán

villagranrodrigo@gmail.com

1 Bariatric Surgery Department, Bupa Antofagasta Clinic, Matta 1868, 2nd floor, Antofagasta, Chile

2 Department of Radiology, Bupa Antofagasta Clinic, Antofagasta, Chile

3 Endocrinology Department, Bupa Antofagasta Clinic, Antofagasta, Chile

4 Faculty of Medicine and Dentistry, Antofagasta University, Antofagasta, Chile patients showed complete recanalization. One patient showed portal cavernomatosis on delayed images. Two patients had a positive thrombophilia test. No portal hypertension endoscopic findings were observed.

Conclusions PMVT is a rare complication, for which smoking was identified as a predominant risk factor. Early diagnosis and prompt anticoagulant therapy could lead to a dramatic decrease in the incidence of intestinal infarction, mortality, and extrahepatic portal hypertension in the near future. However, careful follow-up is necessary to evaluate the impact of PMVT on long-term patient outcomes.

Keywords Portal thrombosis - Splenic thrombosis - Bariatric complications $\cdot$ Sleeve gastrectomy $\cdot$ Thrombophilia .

Bariatric thrombosis

\section{Introduction}

Portomesenteric vein thrombosis (PMVT) is an uncommon but potentially catastrophic clinical complication that may lead to intestinal ischemia and infarction $[1,2]$. Potential etiologies for thrombosis include predisposing factors such as surgery or intra-abdominal inflammation, blunt abdominal trauma, hypercoagulable states, portal hypertension, oral contraceptive use, and bowel obstruction, among others [1-3].

Patients undergoing bariatric surgery are at an increased risk for venous thromboembolism (VTE) due to the underlying inflammatory and hypercoagulable states and because metabolic syndrome may predispose patients to VTE $[4,5]$. PMVT has been observed after surgical procedures, including liver transplantation, splenectomy, and bariatric surgery [6-8]. Although significant progress has been made in bariatric surgery and it is becoming more commonly performed due its 
benefits, it is not exempt from complications that can lead to high morbidity and mortality $[9,10]$.

During a literature search, we identified a limited number of case reports and series describing PMVT following laparoscopic bariatric surgery as an uncommon complication [11-31].

\section{Material and Methods}

This is a retrospective study of a prospectively collected database of patients who underwent LSG for morbid obesity and developed PMVT as a procedure-related complication. Surgery was performed at the Obesity Surgery Department, Bupa Antofagasta Clinic, Antofagasta, Chile, between November 2009 and July 2015.

The following variables were studied: age, gender, body mass index (BMI), thrombosis risk factors, surgical technique, thromboembolism prophylaxis, primary surgery details, PMVT clinical features, diagnosis methods, treatment, and follow-up findings, including thrombophilia testing results, imaging, and endoscopic findings.

\section{Surgical Technique}

We performed 5 incisions; pneumoperitoneum was created using the closed access technique and a Veress needle, with a maximum preset pressure of $15 \mathrm{mmHg}$. A liver retractor was placed through a 5-mm incision in the epigastrium. The gastrosplenic ligament as well as gastrocolic omentum were divided using a vessel sealer and divider (LigaSure Atlas®; Covidien Ltd., Norwalk, CT, USA). To calibrate the gastrectomy, a $34 \mathrm{Fr}$ bougie was used. Gastric resection was performed using a gastrointestinal flexible endo-stapler (EndoGIA Roticulator ${ }^{\circledR}$; Covidien Ltd., Norwalk, CT, USA). A bioabsorbable staple line reinforcement was routinely used (Seamguard® W.L. Gore \& Associates, Flagstaff, AZ, USA), and additional clips were placed to control persistent bleeding. The resected stomach was removed through the supraumbilical port site, and we did not routinely use a retrieval bag.

\section{Multimodal Thromboembolism Prophylaxis Protocol}

Intermittent sequential pneumatic compression (ISPC) was used on inferior limbs, starting with the induction of anesthesia and continuing up to $24 \mathrm{~h}$ postoperatively. Graduated compression stockings (GCSs) were used beginning after hospital admission and continuing during the entire hospitalization, and they were recommended for 7 days after discharge. Treatment with the low-molecular-weight heparin (LMWH) enoxaparin (Clexane®; Sanofi-Aventis, Paris, France) at $40 \mathrm{mg}$ per day was initiated intraoperatively and continued for 7 days. In January 2015, we modified our protocol in an attempt to reduce complication rates; thereafter, LMWH therapy was extended for up to 14 days. Patients start walking within 4-6 h of surgery with assistance.

In all of the patients who developed PMVT, we conducted follow-up computed tomography (CT). Additionally, upper gastrointestinal endoscopy (UGE) was performed to identify signs of portal hypertension.

The thrombophilia test was performed remotely at initial presentation and at least 2-3 weeks after the cessation of anticoagulation therapy [13].

The institutional review board in our hospital approved this study.

\section{Results}

Among the 1578 laparoscopic bariatric surgeries performed, $1236(78 \%)$ patients underwent LSG between November 2005 and July 2015 at the Bupa Antofagasta Clinic; five $(0.4 \%)$ of these patients developed PMVT. The mean age was 34.4 years (range $27-43$ years). Three patients were women. The mean BMI was $38.5 \mathrm{~kg} / \mathrm{m}^{2}$ (range 36.1 $40.9 \mathrm{~kg} / \mathrm{m}^{2}$ ). Four patients had a history of smoking (smoking cessation was instructed at least 6 weeks preoperatively), two used hormonal contraceptives, and none had a history of previous thrombosis or family thrombophilia records. No patient had a history of liver cirrhosis. The patient characteristics are summarized in Table 1.

Table 1 Patient's characteristics and first procedure details

\begin{tabular}{llllllll}
\hline Case & Age & Sex & BMI & Comorbidities & $\begin{array}{l}\text { Hormonal } \\
\text { contraceptive }\end{array}$ & $\begin{array}{l}\text { Smoking } \\
\text { history }\end{array}$ & $\begin{array}{l}\text { Thrombosis history or } \\
\text { thrombophilia family records }\end{array}$ \\
\hline 1 & 43 & F & 37.4 & HTN, IR, FL & Oral & $(-)$ & $(-)$ \\
2 & 37 & F & 36.1 & IFG, IR, FL, DLP & Subdermal implant & $(+)$ & $(-)$ \\
3 & 27 & F & 39.0 & IFG, IR, FL, DLP & No & $(+)$ & $(-)$ \\
4 & 29 & M & 39.1 & HU, IR, DLP & n/a & $(+)$ & $(-)$ \\
5 & 36 & M & 40.9 & IFG, IR, FL, DLP, OSA & n/a & $(+)$ & $(-)$
\end{tabular}

$F$ female, $M$ male, $H T N$ hypertension, $I R$ insulin resistance, $F L$ fatty liver, $I F G$ impaired fasting glucose, $D L P$ dyslipidemia, $H U$ hyperuricemia, $O S A$ obstructive sleep apnea, n/a not applicable 
Table 2 First procedure and stay details

\begin{tabular}{|c|c|c|c|c|c|c|}
\hline Case & LMWH enoxaparin doses & LMWH 1st doses & LMWH length (days) & OR time (min) & Liver retractor & LOS (hours) \\
\hline 1 & $40 \mathrm{mg} /$ day & Anesthesia induction & 7 & 90 & Nathanson & 72 \\
\hline 2 & $40 \mathrm{mg} /$ day & Anesthesia induction & 7 & 60 & Nathanson & 72 \\
\hline 3 & $40 \mathrm{mg} /$ day & Anesthesia induction & 7 & 105 & Nathanson & 48 \\
\hline 4 & $40 \mathrm{mg} /$ day & Intraoperatory & 7 & 75 & Nathanson & 48 \\
\hline 5 & $40 \mathrm{mg} /$ day & Intraoperatory & 14 & 135 & No & 48 \\
\hline
\end{tabular}

LMWH low-molecular-weight heparin, OR operation room, LOS length of stay

During the LSG procedure, no complications were observed, and no conversion to open surgery was needed. The mean operation room time was $93 \mathrm{~min}$ (range 60-135 min). A liver retractor was not necessary in one patient due to the patient's liver anatomy. All of the patients received complete thromboembolic prophylaxis. One of the patients who developed PMVT received 14 days of treatment due to improvements in the thromboembolic prophylaxis protocol. All of the patients were routinely encouraged to walk with assistance early after surgery; intravenous fluid therapy was also administered. No complications were observed during hospitalization. All of the patients were discharged $48-72 \mathrm{~h}$ after surgery.
The first procedure and duration details are summarized in Table 2.

The patients were readmitted at a median of 20 days (range 11-50 days) postoperatively. The main symptom was abdominal pain in all of our patients, starting at 3.4 days (range 25 days) before emergency room consultation. Four of five patients showed elevated inflammatory parameters such as the white blood cell count (WBC) and C-reactive protein (CRP) levels at diagnosis. All of the cases were diagnosed by abdominal and pelvic CT enhanced with oral and intravenous contrast according to the emergency unit flowchart for bariatric readmission patients. The patients were admitted to
Table 3 Portal thrombosis symptoms, readmission, and treatment. Follow-up with upper gastroscopy and thrombophilia screening

\begin{tabular}{llllll}
\hline Patient & 1 & 2 & 3 & 4 & 5 \\
\hline Symptoms: F: fever, N: nauseas Pain location & $\mathrm{F}(-)$ & $\mathrm{F}(-)$ & $\mathrm{F}(+)$ & $\mathrm{F}(-)$ & $\mathrm{F}(-)$ \\
& $\mathrm{N}(-)$ & $\mathrm{N}(-)$ & $\mathrm{N}(-)$ & $\mathrm{N}(-)$ & $\mathrm{N}(+)$ \\
& $\mathrm{RL}, \mathrm{RH}$ & $\mathrm{RH}$ & $\mathrm{LL}$ & $\mathrm{E}$ & $\mathrm{E}$ \\
Start symptoms before readmission & 5 & 2 & 3 & 4 & 3 \\
POD Readmission & 15 & 50 & 11 & 12 & 16 \\
WBC count $\left(\times 10^{3} / \mathrm{mm} 3\right)$ & 6.9 & 5.2 & 15.7 & 12.1 & 14.7 \\
C-reactive protein (mg/L) & 81.9 & 1 & 215.3 & 199.6 & 61.7 \\
Hematocrit (\%) & 43.8 & 39.3 & 41.9 & 45.7 & 46.3 \\
Hemoglobin (g/dL) & 15.2 & 13.3 & 14.1 & 16.0 & 15.9 \\
Coagulation tests PT/ & $12.2 /$ & $12.4 /$ & $13.5 /$ & $13.6 /$ & $12.3 /$ \\
APTT (seg) & 27.2 & 53.6 & 28.5 & 33.6 & 28.2 \\
AST/ALT levels (U/L) & $61 /$ & $9 /$ & $13 /$ & $23 /$ & $29 /$ \\
& 120 & 12 & 19 & 33 & 86 \\
Bilirubin levels & $0.6 /$ & $0.7 /$ & $0.2 /$ & $1 /$ & $0.3 /$ \\
Unconjugated/conjugated (mg/dL) & 0.6 & 0.3 & 0.2 & 0.7 & 0.2 \\
Hours at remission of symptoms & 96 & 48 & 48 & 72 & 48 \\
Anticoagulant Therapy & Ac & Ac & Wf & Ac & Wf \\
Length of stay (days) & 7 & 8 & 10 & 6 & 7 \\
Anticoagulant therapy length (months) & Indefinitely & 4 & 6 & 6 & 6 \\
Esophageal varices at UGE & $(-)$ & $(-)$ & $(-)$ & $(-)$ & $(-)$ \\
(POD, months) & 22 & 12 & 25 & 17 & 2 \\
Thrombophilia screening & ProtC def & $\mathrm{n} / \mathrm{a}$ & $\mathrm{n} / \mathrm{a}$ & $\mathrm{ProtC}$ def & $\mathrm{Neg}$ \\
\hline
\end{tabular}

$F$ fever, $N$ nauseas, $R L$ right lumbar, $R H$ right hypochondrial, $L L$ left lumbar, $E$ epigastric, $P O D$ postoperative day, $A S T$ aspartate transaminase, ALT alanine transaminase, $P T$ prothrombin time, APTT activated partial thromboplastin time, $A c$ acenocoumarol, $W f$ warfarin, def deficit, $N e g$ negative, $U G E$ upper gastrointestinal endoscopy, $n / a$ not available 
the intensive care unit and immediately underwent intravenous unfractionated heparin therapy, followed by strict medical and CT or ultrasound follow-up, considering the high risk of thrombosis progression to mesenteric veins and small bowel infarction. The therapy was successful, and surgical exploration was not needed. The patients showed satisfactory recovery and remission of the symptoms after $62.4 \mathrm{~h}$ (range 48$96 \mathrm{~h}$ ), and oral intake was progressively resumed. All of the patients were discharged without symptoms after a mean hospitalization period of 7.6 days (range 6-10 days). Anticoagulation therapy with acenocoumarol or warfarin was started after 3.5 days (range $2-5$ days) and continued for 4-6 months. One patient continued long-term therapy. The clinical features and treatment details of PMVT are summarized in Table 3.

The partial or complete thrombosis location of the portal vein and its branches were identified (Fig. 1), and all of the patients presented with partial or complete extension to the superior mesenteric vein. Two of the cases even progressed to complete obstruction, although none of them presented with intestinal infarction; those cases showed important development of the collateral vessels. Three patients presented with concomitant thrombosis of the splenic vein. Thrombophilia

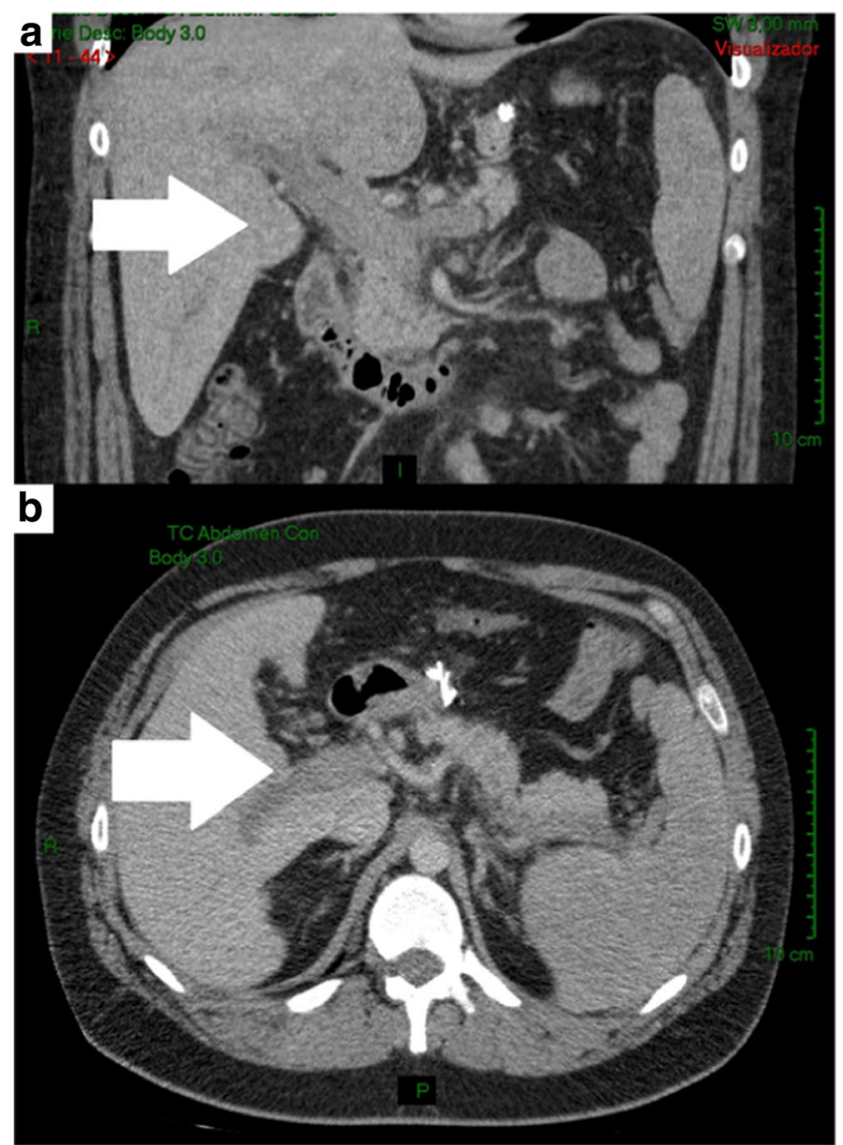

Fig. 1 Complete portal thrombosis. CT scan at readmission. a Coronal section and $\mathbf{b}$ axial sections at 12 postoperative day evaluation was achieved in 3 patients ( 2 refused to undergo the procedure) 6 weeks after anticoagulant therapy was ended (as suggested by several guidelines). Two patients showed a protein $\mathrm{C}$ deficiency.

After the discharge of patients who developed PMVT, follow-up was conducted using CT and UGE. Two patients showed complete recanalization at the thrombosis site, and three others showed partial recanalization at different degrees. One patient displayed portal cavernomatosis (Fig. 2). No portal hypertension endoscopic findings were observed at a median follow-up of 36 months. The CT images at readmission and follow-up are summarized in Table 4.

\section{Discussion}

The development of venous thrombosis is generally considered to involve a combination of locoregional and systemic prothrombogenic factors $[32,33]$.

The etiology of PMVT complication after laparoscopic surgery is likely multifactorial. Some of the factors that may contribute to the pathogenesis of this condition include undiagnosed thrombophilia, local injury near the portal flow, surgical damage (including direct trauma leading to diminished blood flow), oral contraceptive use, increased intra-abdominal pressure due to pneumoperitoneum, [14, 16, 20, 26, 34-39] and prolonged time in the reverse Trendelenburg position [18].

PMVT has been well described as a post-splenectomy complication due to hematologic causes, and the symptomatic PVT incidence may vary from 0.2 to $0.9 \%$, with an increased incidence of up to $10.9 \%$ in asymptomatic patients $[19,40]$.

In the bariatric field, surgery-related PMVT has been described as an infrequent complication in several case reports and series [11-31]. Multiples hypotheses have been put forward to explain this complication. In this particular group of patients, we included the possible etiologic factors of

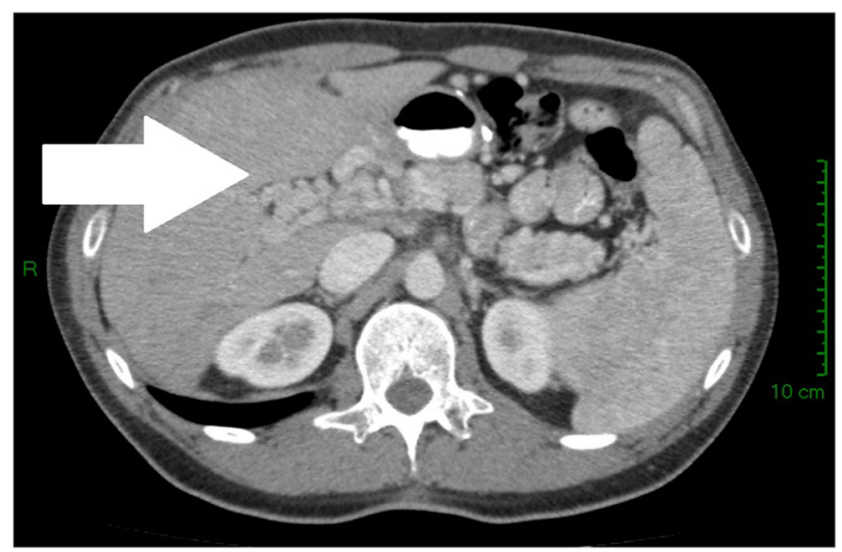

Fig. 2 Portal cavernomatosis. CT scan axial section at 20 months after PMVT 
Table 4 CT scan images at readmission and follow-up

\begin{tabular}{|c|c|c|c|c|c|c|c|c|c|}
\hline \multirow[t]{2}{*}{ Case } & \multirow[t]{2}{*}{ CT POD (days) } & \multicolumn{4}{|l|}{ iPV } & \multirow[t]{2}{*}{ ePV } & \multirow[t]{2}{*}{ SMV } & \multirow[t]{2}{*}{$\mathrm{pSV}$} & \multirow[t]{2}{*}{ dSV } \\
\hline & & LPV & RPV & $\mathrm{RaPV}$ & $\mathrm{RpPV}$ & & & & \\
\hline \multirow[t]{4}{*}{1} & 15 & $(-)$ & $\mathrm{T}$ & $\mathrm{T}$ & $\mathrm{T}$ & $\mathrm{Pa} 75$ & $\mathrm{~Pa} 75$ & $(-)$ & $(-)$ \\
\hline & 22 & $(-)$ & $\mathrm{T}$ & $\mathrm{T}$ & $\mathrm{T}$ & $\mathrm{Pa} 30$ & $\mathrm{~Pa} 30$ & $(-)$ & $(-)$ \\
\hline & 29 & $(-)$ & $(-)$ & $(-)$ & $(-)$ & $(-)$ & $(-)$ & $(-)$ & $(-)$ \\
\hline & 842 & $(-)$ & $(-)$ & $(-)$ & $(-)$ & $(-)$ & $(-)$ & $(-)$ & $(-)$ \\
\hline \multirow[t]{3}{*}{2} & 50 & $\mathrm{~Pa} 30$ & $\mathrm{~T}$ & $\mathrm{~T}$ & $\mathrm{~T}$ & $\mathrm{~Pa} 75$ & $\mathrm{~Pa} 75$ & $(-)$ & $(-)$ \\
\hline & 93 & $(-)$ & $\mathrm{T}$ & $\mathrm{T}$ & $\mathrm{T}$ & $\mathrm{Pa} 30$ & $\mathrm{~Pa} 30$ & $(-)$ & $(-)$ \\
\hline & 595 & $(-)$ & $(-)$ & $\mathrm{T}$ & $\mathrm{T}$ & $(-)$ & $(-)$ & $(-)$ & $(-)$ \\
\hline \multirow[t]{4}{*}{3} & 11 & $(-)$ & $\mathrm{T}$ & $\mathrm{T}$ & $\mathrm{T}$ & $\mathrm{Pa} 50$ & $\mathrm{~Pa} 30$ & $\mathrm{~Pa} 75$ & $\mathrm{~T}$ \\
\hline & 15 & $(-)$ & $\mathrm{T}$ & $\mathrm{T}$ & $\mathrm{T}$ & $\mathrm{Pa} 50$ & $\mathrm{~Pa} 30$ & $\mathrm{~Pa} 75$ & $\mathrm{~T}$ \\
\hline & 21 & $(-)$ & $\mathrm{T}$ & $\mathrm{T}$ & $\mathrm{T}$ & $\mathrm{Pa} 50$ & $\mathrm{~Pa} 30$ & $\mathrm{~Pa} 75$ & $\mathrm{~T}$ \\
\hline & 747 & $(-)$ & $(-)$ & Pa50 & $\mathrm{T}^{\mathrm{b}}$ & $(-)$ & $(-)$ & $\mathrm{Pa30w/coll}$ & $\mathrm{Pa} 30 \mathrm{w} / \mathrm{coll}$ \\
\hline \multirow[t]{3}{*}{4} & 12 & $\mathrm{~T}$ & $\mathrm{~T}$ & $\mathrm{~T}$ & $\mathrm{~T}$ & $\mathrm{~T}$ & $\mathrm{~T}$ & $\mathrm{~T}$ & $\mathrm{~T}$ \\
\hline & 17 & $\mathrm{~T}$ & $\mathrm{~T}$ & $\mathrm{~T}$ & $\mathrm{~T}$ & $\mathrm{~T}$ & $\mathrm{~T}$ & $\mathrm{~T}$ & $\mathrm{~T}$ \\
\hline & 610 & $(-)$ & $(-)$ & $(-)$ & $(-)$ & Cavernomatosis & Tw/coll & $\mathrm{Pa} 80 \mathrm{w} /$ coll & $\mathrm{Pa} 80$ w/coll \\
\hline \multirow[t]{4}{*}{5} & 16 & $\mathrm{~T}$ & $\mathrm{~T}$ & $\mathrm{~T}$ & $\mathrm{~T}$ & $\mathrm{~T}$ & $\mathrm{~T}$ & $\mathrm{~T}$ & $\mathrm{~T}$ \\
\hline & 20 & $\mathrm{~T}$ & $\mathrm{~T}$ & $\mathrm{~T}$ & $\mathrm{~T}$ & $\mathrm{~T}$ & $\mathrm{~T}$ & $\mathrm{~T}$ & $\mathrm{~T}$ \\
\hline & 60 & $\mathrm{~T}$ & $\mathrm{~T}$ & $\mathrm{~Pa} 50$ & $\mathrm{~T}$ & $\mathrm{~Pa} 30$ & Tw/coll & Tw/coll & Tw/coll \\
\hline & 89 & $(-)$ & $(-)$ & $(-)$ & $(-)$ & $(-)$ & Tw/coll & Tw/coll & Tw/coll \\
\hline
\end{tabular}

$P O D$ postoperative day, $T>90 \%$ thrombosis, $P a(x)$ partial thrombosis percentage of lumen occlusion was calculated by the area of thrombus divided by the area of vein lumen, for more than $90 \%$ was defined as complete, (-) permeable, $w /$ coll with collaterals, $i P V$ intrahepatic portal vein, $L P V$ left portal vein, $R P V$ right portal vein, $R a P V$ right anterior portal vein, $R p P V$ right posterior portal vein, $e P V$ extrahepatic portal vein, $S M V$ superior mesenteric vein, $p S V$ proximal splenic vein, $d S V$ distal splenic vein

${ }^{a}$ CT scan report: segmental portal vein branches according to Couinaud's classification

${ }^{\mathrm{b}}$ Atrophy of the posterior segment VI

metabolic syndrome and the thromboembolic risk of obesity itself. In a systematic review, Rocha et al. reported the association between VTE and obese patients undergoing bariatric surgery with an incidence of deep vein thrombosis (DVT) and pulmonary embolism (PE) after surgery varying between 1.2 to $1.6 \%$ and 0.8 to $3.2 \%$, respectively [39]. Nevertheless, PMVT seems to occur more frequently in patients undergoing LSG than in those undergoing other bariatric procedures.

Specifically, during the LSG procedure, the following contributing factors are considered the most significant:

a. Mechanical or thermal effect on the left gastroepiploic arcade or short vessels during the skeletonization of the greater curvature [11]. Intraoperative surgical manipulation may damage the splanchnic endothelium and lead to local thrombus formation that may then propagate throughout the portal venous system [18].

b. Blood flow pattern change and diminished flow due to ligation of the short gastric and tributary vessels $[18,20]$.

c. Splenic vein direct contact. Direct contact with the splenic or superior mesenteric vein (SMV) was proposed to be able to occur during surgery and cause thrombosis [11, 26]. d. Splenic ischemia or infarction. The ligation of the short vessels during LSG may lead to insufficient perfusion of the upper pole of the spleen, perceptible as a demarcation during the operation. Such a condition, while asymptomatic in most cases, may occasionally lead to the symptomatic development of a splenic infarct and even subsequent abscess, reflecting the release of inflammatory mediators [41-44].

e. Dehydration. Liquid intake is limited after bariatric surgery due to the restrictive component of these surgeries, and some patients may be in a negative fluid balance after discharge, which predisposes them to VTE (including PMVT) [11, 20, 26].

The liver retractor used during LSG may cause blood stasis within the liver; the presence of a retrograde thrombosis might be another underlying factor for PMVT [11, 36, 37]. In our series, there was one patient for whom the liver retractor was not used due to anatomic properties such as a small left lobe, and this patient developed PMVT without major liver manipulation during the procedure. Goitien et al., in a multicenter study including 5706 bariatric surgeries, reported $17(0.3 \%)$ cases of PMVT; all cases but one occurred after LSG, and no 
PMVT occurred in the group of approximately 1000 patients who had undergone laparoscopic Roux-en-Y gastric bypass (LRYGB) [20]. Additionally, in our complete series of 1578 surgeries, no PMVT was reported outside of the LSG group.

The broad spectrum of clinical presentations of PMVT ranges from incidental findings, in an asymptomatic patient, to life-threatening bowel infarction [13, 14, 17]. Leukocytosis and mild elevation of liver function tests are also observed. Thus, physical examination findings can be normal, or alternatively, if associated with bowel ischemia, patients could present with peritonitis and septic shock [18]. CT enhanced with oral and intravenous contrast has been reported in published studies to diagnose and monitor the patient's course with a sensitivity of $90 \%[13,14]$.

Once a diagnosis of PMVT is made, treatment should be promptly started. In patients presenting with peritonitis or shock, an exploratory laparotomy is required with possible resection of necrotic bowel. Full anticoagulation with either subcutaneous LMWH or intravenous unfractionated heparin is applied in patients who do not develop bowel ischemia or necrosis $[19,45]$. In patients with recent portal or mesenteric vein thrombosis treated with anticoagulation, not only the follow-up imaging data but also the clinical outcome appears favorable [46]. This treatment is continued and changed to oral anticoagulation (target international normalized ratio, 2.5-3), which should be continued for several months; the duration of this treatment will depend on the coagulation profiles and a hematologic consultation [19, 46].

Early diagnosis and treatment of the underlying causal factors (abdominal sepsis and pro-thrombotic factors) following prompt anticoagulant therapy could lead to a dramatic decrease in the incidence of extrahepatic portal hypertension in the near future [46, 47]. In contrast, late detection and treatment may result in pre-sinusoidal portal hypertension with the associated sequelae and portal cavernomatosis; the latter can result in portal hypertension and complications such as variceal gastrointestinal bleeding [21].

Prophylaxis is routinely perioperatively administered as a standard prevention measure for DVT in most bariatric centers, and many different regimens are used regarding the time of initiation (pre-surgery, anesthesia induction, intraoperatively, and post-surgery), specific pharmacologic agent used, dosage, and length of treatment (during hospital stay until discharge and up to 2 weeks after surgery) $[11,15,17,18,20$, 22, 23, 26-31]. However, there is no consensus concerning PMVT prophylaxis.

\section{Conclusions}

PMVT is a rare complication after laparoscopic bariatric surgery, as observed in our series.
A history of smoking was a predominant risk factor for PMVT, and abdominal pain was the main symptom. CT of the abdomen and pelvis is the proven method of choice for PMVT diagnosis. Early diagnosis and appropriate management are essential in preventing catastrophic complications such as intestinal infarction and corresponding increases in morbidity and mortality. Anticoagulant therapy is generally satisfactory to avoid thrombosis progression and to achieve partial or complete recanalization. However, careful follow-up is necessary to evaluate the impact of PMVT on long-term patient outcomes.

Acknowledgments The authors have no relevant financial or nonfinancial relationships to disclose.

\section{Compliance with Ethical Standards}

Conflict of Interest The authors declare that they have no conflicts of interest.

Ethical Statement All procedures performed in studies involving human participants were in accordance with the ethical standards of the Institutional Review Board and with the 1964 Helsinki declaration and its later amendments or comparable ethical standards.

This is a retrospective study; for this type of study, formal consent is not required.

Informed Consent Informed consent was obtained from all individual participants included in the study.

Open Access This article is distributed under the terms of the Creative Commons Attribution 4.0 International License (http:// creativecommons.org/licenses/by/4.0/), which permits unrestricted use, distribution, and reproduction in any medium, provided you give appropriate credit to the original author(s) and the source, provide a link to the Creative Commons license, and indicate if changes were made.

\section{References}

1. Ögren M, Bergqvist D, Björck M, et al. Portal vein thrombosis: prevalence, patient characteristics and lifetime risk: a population study based on 23,796 consecutive autopsies. World J Gastroenterol. 2006;12(13):2115-9.

2. Valla D, Condat B. Portal vein thrombosis in adults: pathophysiology, pathogenesis and management. J Hepatol. 2000;32(5):865-71.

3. Abdollahi M, Cushman M, Rosendaal FR. Obesity: risk of venous thrombosis and the interaction with coagulation factor levels and oral contraceptive use. Thromb Haemost. 2003;89(3):493-8.

4. Samama MM. An epidemiologic study of risk factors for deep vein thrombosis in medical outpatients: the Sirius study. Arch Intern Med. 2000;160(22):3415-20.

5. Hansson PO, Eriksson H, Welin L, et al. Smoking and abdominal obesity: risk factors for venous thromboembolism among middleaged men: "the study of men born in 1913.". Arch Intern Med. 1999;159(16):1886-90.

6. Ikeda M, Sekimoto M, Takiguchi S, et al. High incidence of thrombosis of the portal venous system after laparoscopic splenectomy: a prospective study with contrast-enhanced CT scan. Ann Surg. 2005;241(2):208-16. 
7. Stella M, Serventi A, Friedman D. Right portal vein thrombosis after splenectomy for trauma. J Gastrointest Surg. 2005;9(5):646-7.

8. Yerdel MA, Gunson B, Mirza D, et al. Portal vein thrombosis in adults undergoing liver transplantation: risk factors, screening, management, and outcome. Transplantation. 2000;69(9):1873-81.

9. Moon Han S, Kim WW, Oh JH. Results of laparoscopic sleeve gastrectomy (LSG) at 1 year in morbidly obese Korean patients. Obes Surg. 2005;15(10):1469-75.

10. Lee CM, Cirangle PT, Jossart GH. Vertical gastrectomy for morbid obesity in 216 patients: report of two-year results. Surg Endosc. 2007;21(10):1810-6.

11. Salinas J, Barros D, Salgado N, et al. Portomesenteric vein thrombosis after laparoscopic sleeve gastrectomy. Surg Endosc. 2014;28(4):1083-9.

12. Denne JL, Kowalski C. Portal vein thrombosis after laparoscopic gastric bypass. Obes Surg. 2005;15(6):886-9.

13. Johnson CM, de la Torre RA, Scott JS, et al. Mesenteric venous thrombosis after laparoscopic roux-en-Y gastric bypass. Surg Obes Relat Dis. 2005;1(6):580-2. Discussion:582-3.

14. Swartz DE, Felix EL. Acute mesenteric venous thrombosis following laparoscopic roux-en-Y gastric bypass. JSLS. 2004;8(2):165-9.

15. Sonpal IM, Patterson L, Schreiber H, et al. Mesenteric venous thrombosis after gastric bypass. Obes Surg. 2004;14(3):419-21.

16. Bellanger DE, Hargroder AG, Greenway FL. Mesenteric venous thrombosis after laparoscopic sleeve gastrectomy. Surg Obes Relat Dis. 2010;6(1):109-11.

17. Singh P, Sharma M, Gandhi K, et al. Acute mesenteric vein thrombosis after laparoscopic gastric sleeve surgery for morbid obesity. Surg Obes Relat Dis. 2010;6(1):107-8.

18. James AW, Rabl C, Westphalen AC, et al. Portomesenteric venous thrombosis after laparoscopic surgery: a systematic literature review. Arch Surg. 2009;144(6):520-6.

19. van't Riet M, Burger JW, van Muiswinkel JM, et al. Diagnosis and treatment of portal vein thrombosis following splenectomy. Br J Surg. 2000;87(9):1229-33.

20. Goitein D, Matter I, Raziel A, et al. Portomesenteric thrombosis following laparoscopic bariatric surgery: incidence, patterns of clinical presentation, and etiology in a bariatric patient population. JAMA Surg. 2013;148(4):340-6.

21. Plessier A, Darwish-Murad S, Hernandez-Guerra M, et al. Acute portal vein thrombosis unrelated to cirrhosis: a prospective multicenter follow-up study. Hepatology. 2010;51(1):210-8.

22. Calmes JM, Bettschart V, Raffoul W, et al. Band infection with splenoportal venous thrombosis: an unusual but severe complication of gastric banding. Obes Surg. 2002;12(5):699-702.

23. Cesaretti M, Elghadban H, Scopinaro N, et al. Portomesenteric venous thrombosis: an early postoperative complication after laparoscopic biliopancreatic diversion. World J Gastroenterol. 2015;21(8):2546-9.

24. Carlin A, Finks J, Birkmeyer N, et al. An unintended consequence of sleeve gastrectomy: portomesenteric venous thrombosis. Surg Obes Relat Dis. 2015;11(6):S31.

25. Surve A, Cottam D, Zaveri H, et al. Portal mesenteric vein thrombosis after laparoscopic sleeve gastrectomy: clinical presentation and management. Surg Obes Relat Dis. 2015;11(6):S107.

26. Rottenstreich A, Khalaileh A, Elazary R. Sleeve gastrectomy and mesenteric venous thrombosis: report of 3 patients and review of the literature. Surg Obes Relat Dis. 2014;10(6):e57-61.

27. Keung CH, Gander JW, Zitsman JL. Mesenteric venous thrombosis following vertical sleeve gastrectomy in an adolescent. Surg Obes Relat Dis. 2015;11(2):e23-6.
28. Roy P, De A. Liver and bowel infarction secondary to portomesenteric vein thrombosis following laparoscopic sleeve gastrectomy. Saudi J Obes. 2015;3:29-31.

29. Darcy DG, Charafeddine AH, Choi J, et al. Portomesenteric vein thrombosis, bowel gangrene, and bilateral pulmonary artery embolism two weeks after laparoscopic sleeve gastrectomy. Case Rep Surg. 2015;2015:705610.

30. Berthet B, Bollon E, Valero R, et al. Portal vein thrombosis due to factor 2 Leiden in the post-operative course of a laparoscopic sleeve gastrectomy for morbid obesity. Obes Surg. 2009;19(10):1464-7.

31. Rosenberg JM, Tedesco M, Yao DC, et al. Portal vein thrombosis following laparoscopic sleeve gastrectomy for morbid obesity. JSLS. 2012;16(4):639-43.

32. Denninger MH, Chait Y, Casadevall N, et al. Cause of portal or hepatic venous thrombosis in adults: the role of multiple concurrent factors. Hepatology. 2000;31(3):587-91.

33. Rosendaal FR. Venous thrombosis: a multicausal disease. Lancet 1999;353(9159):1167-73.

34. Dentali F, Romualdi E, Ageno W. The metabolic syndrome and the risk of thrombosis. Haematologica. 2007;92(9):297-9.

35. Takagi $\mathrm{S}$. Hepatic and portal vein blood flow during carbon dioxide pneumoperitoneum for laparoscopic hepatectomy. Surg Endosc. 1998;12(5):427-31.

36. Jakimowicz J, Stultiëns G, Smulders F. Laparoscopic insufflation of the abdomen reduces portal venous flow. Surg Endosc. 1998;12(2): 129-32.

37. Ishizaki Y, Bandai Y, Shimomura K, et al. Changes in splanchnic blood flow and cardiovascular effects following peritoneal insufflation of carbon dioxide. Surg Endosc. 1993;7(5):420-3.

38. Lindberg F. Carbon dioxide pneumoperitoneum - hemodynamic consequences and thromboembolic complications [Dissertation]. Comprehensive summaries of Uppsala dissertations from the Faculty of Medicine; 2002.

39. Rocha AT, de Vasconcellos AG, da Luz Neto ER, et al. Risk of venous thromboembolism and efficacy of thromboprophylaxis in hospitalized obese medical patients and in obese patients undergoing bariatric surgery. Obes Surg. 2006;16(12):1645-55.

40. Parker 3rd HH, Bynoe RP, Nottingham JM. Thrombosis of the portal venous system after splenectomy for trauma. J Trauma. 2003;54(1):193-6.

41. Dhanasopon AP, Lewis CE, Folek JM, et al. Splenic infarct as complication of sleeve gastrectomy. Surg Obes Relat Dis. 2009;5(5):626-9.

42. Stamou KM, Menenakos E, Gomatos IP, et al. Clinical implications of sleeve gastrectomy as a source of spleen infarction or ischemia. Obes Surg. 2011;21(10):1490-3.

43. Chaudhry UI, Mikami DJ. The perfect storm: a foreign body and splenic infarct mimicking a sleeve gastrectomy leak. Surg Obes Relat Dis. 2014;10(6):e63-6.

44. Iannelli A, Schneck A, Gugenheim J. Segmental splenic ischemia after laparoscopic sleeve gastrectomy. Surg Obes Relat Dis. 2015;11(1):265-6.

45. Joh JH, Kim DI. Mesenteric and portal vein thrombosis: treated with early initiation of anticoagulation. Eur J Vasc Endovasc Surg. 2005;29:204-8.

46. Condat B, Pessione F, Helene Denninger M, et al. Recent portal or mesenteric venous thrombosis: increased recognition and frequent recanalization on anticoagulant therapy. Hepatology. 2000;32(3): 466-70.

47. Kumar S, Sarr MG, Kamath PS. Mesenteric venous thrombosis. N Engl J Med. 2001;345(23):1683-8. 